\title{
Alimentación y reproducción de Creagrutus brevipinnis (Pisces: Characidae) en Alto Cauca, Colombia
}

\author{
César Román-Valencia \\ 1 Universidad del Quindío, Depto. de Biología, A.A. 460, Armenia, Colombia. Correo electrónico: croman@ cocora.uniquin- \\ dio.edu.co
}

\author{
Recibido 18-VIII-1997. Corregido 27-V-1998. Aceptado 8-VI-1998.
}

\begin{abstract}
Stomach contents and reproduction were studied in characid fish (C. brevipinnis)collected in the Vieja River Basin, Alto Cauca, Colombia (July 1995 and December 1996); the temperature in the region was $21.7^{\circ} \mathrm{C}(18.70$ $\left.24.40^{\circ} \mathrm{C}\right), \mathrm{pH}$ was neutral and water oxygen content high $(8 \mathrm{ppm}) ; C$. brevipinnis is nonmigratory and sympatric with other 30 fish species. The fish is insectivorous and specializes on Diptera (Simuliidae) and Ephemeroptera; being a third level predator in the trophic web; it feeds from 06:00-18:00 hr ; the results indicate seasonal variation in feeding activity $(\mathrm{F}=19.04 \mathrm{P}<0.0001)$ and a negative correlation between stomach weight and voracity indexes; in the reproductive season food in take is low; the ovaries mature primarily between May and January ; fecundity is low (613 oocytes) and the eggs are of small (diameter $0.6254 \mathrm{~mm})$; the sex ratio is $1: 1\left(\mathrm{X}^{2}=2.56 \mathrm{P}<0.1 \mathrm{df}=1\right)$ and the mean size at maturity is $4.0 \mathrm{~cm}$ (standard length).
\end{abstract}

Key words: Trophic, ecology, feeding, reproduction, Creagrutus brevipinnis, tropical fishes.

Observaciones no concluyentes sobre la ecología de las especies de Creagrutus se pueden encontrar en: Saul (1967), Harold et al. (1994), Harold \& Vari (1994) y Vari et al. (1993). En el único trabajo (Ortaz 1992) que analiza la dieta de una especie de Creagrutus, se planteó que $C$. beni del río Limón en la región centro-norte de Venezuela se alimenta predominantemente de Diptera, Trichoptera, Coleoptera, restos de insectos y vegetales. De $C$. brevipinnis, Román-Valencia (1995) expresó observaciones preliminares sobre el ambiente y su dieta, que comprende insectos (Díptera, Hemíptera) y estiércol de ganado vacuno; RomanValencia \& Cala (1997) plantearon que es una especie endémica del Alto Cauca. Por lo tanto, los estudios ecológicos en comunidades (Ortaz
1992) y en poblaciones de alta montaña neotropical son escasos. Mas aún si el estudio de los hábitos alimenticios basados en el análisis de contenido estomacal es uno de los métodos mas usados para el conocimiento del rol trofico de los peces en los ecosistemas acuáticos (Ferriz \& Salas 1996). En esta óptica, el objetivo de este trabajo es establecer aspectos ecológicos y troficos de $C$. brevipinnis a partir de un análisis cualitativo y cuantitativo del contenido gástrico, voracidad, la relación contenido estomacal medio externo, y posibles cambios relacionados con la alimentación; asociaciones entre el peso total, sexo, índice gonosomático, factor de condición; además, obtener datos referentes a la biometría del tracto digestivo, frecuencia de captura, abundancia relativa y algunos parámetros físicos y químicos. 


\section{MATERIALES Y MÉTODOS}

Utilizando red de arrastre se realizaron capturas en ríos, quebradas y arteria principal de la cuenca del río La Vieja (75 10'- 75 50' W y 4 10' $440^{\prime} \mathrm{N}$ ), complementando con algunas colectas en la cuenca del río La Paila entre julio de 1995 y diciembre de 1996. Se examinaron 465 ejemplares adultos (talla promedio $4.0 \mathrm{~cm}$ de longitud ). Se hicieron disecciones de estómagos, gónadas e intestinos. Los parámetros físico-químicos se determinaron in situ entre enero y diciembre de 1996. Las determinaciones de la ictiofauna acompañante se realizaron integralmente in situ y se devolvieron al agua; salvo excepciones, se fijaron en formol al $10 \%$ para su posterior confirmación taxonómica. Ejemplares testigo se hallan depositados en la Unidad de Ictiología del Instituto de Ciencias Naturales, Universidad Nacional de Colombia, Bogotá (ICN-MHN 978-2469) y en el Laboratorio de Biología, Universidad del Quindio, Armenia (IUQ 170). Con los métodos de frecuencia de ocurrencia y numérica (Hyslop 1980) se realizó el análisis de los presentes ítems en 345 estómagos con contenido. El índice de voracidad se determinó a partir del coeficiente (b) de regresión (Movillo \& Bahamonde 1971). La relación gonosomática (Vazzoler 1996) se calculó con la ecuación : RGS $=$ Wo/Wc X 100, siendo $\mathrm{Wc}=\mathrm{Wt}-\mathrm{Wo}$ donde $\mathrm{Wo}=$ peso de la góna$\mathrm{da}, \mathrm{Wt}=$ peso total y $\mathrm{Wc}=$ peso del cuerpo. $\mathrm{El}$ análisis de la actividad alimentaria $(\mathrm{R})$ se basó en el coeficiente de contenido estomacal (Ghazai et al. 1991). El factor de condición total alométrico (Vazzoler 1996) se obtuvo por la ecuación : K= $\mathrm{W} / \mathrm{Ltb}$ donde $\mathrm{W}=$ peso del cuerpo, $\mathrm{Lt}=$ longitud total y $b=0.434$.

Los datos físicos y químicos se tomaron basados en las técnicas descritas por la Apha (Anónimo 1985); tanto la coloración como el tipo de sustrato de los biotopos calificados por observación directa; el oxigeno disuelto y la temperatura se obtuvieron con oximetro, el $\mathrm{pH}$ con potenciometro, la velocidad de la corriente por el tiempo que tarda una bola de icopor en recorrer dos metros sobre la superficie del agua, el ancho y la profundidad en cada sitio de muestreo, medidas con decámetro y una vareta graduada en $\mathrm{cm}$.

\section{RESULTADOS}

Hábitat.- C. brevipinnis habita en la ribera del río La Vieja, de anchura total $46 \mathrm{~m}$, en afluentes de tipo secundario entre los $900 \mathrm{y}$ 1200 m.s.n.m. , de ancho promedio ponderado $7.95 \mathrm{~m}$ y profundidad $0.58 \mathrm{~m}$. Charcos y rápidos forman un gradiente moderado de flujo permanente un sustrato estructurado por arena, piedra y en algunos sitios material orgánica descompuesta o detritos; las riberas de las quebradas están cubiertas de vegetación arbustiva, mientras que al río La Vieja y sus afluentes mayores lo dominan playas de arena, vegetación arbórea con predominio de Guadua angustifolia y pasto kikuyo (Poaceae) para el ganado vacuno. El agua es cristalina o clara, pero después de una lluvia breve de un dia u horas se torna café o gris. La temperatura superficial osciló entre $18.7^{\circ} \mathrm{C}$ (enero) y $24.4^{\circ} \mathrm{C}$ (junio); el oxígeno disuelto varió entre $6.8 \mathrm{mg} / \mathrm{l}$ (mayo y octubre) y $8.4 \mathrm{mg} / \mathrm{l}$ (agosto) y el pH estuvo alrededor de la neutralidad (Fig. 1); la profundidad osciló de $0.5 \mathrm{~m}$ ( enero, marzo, mayo y diciembre) a 0.72 $\mathrm{m}$ (abril).

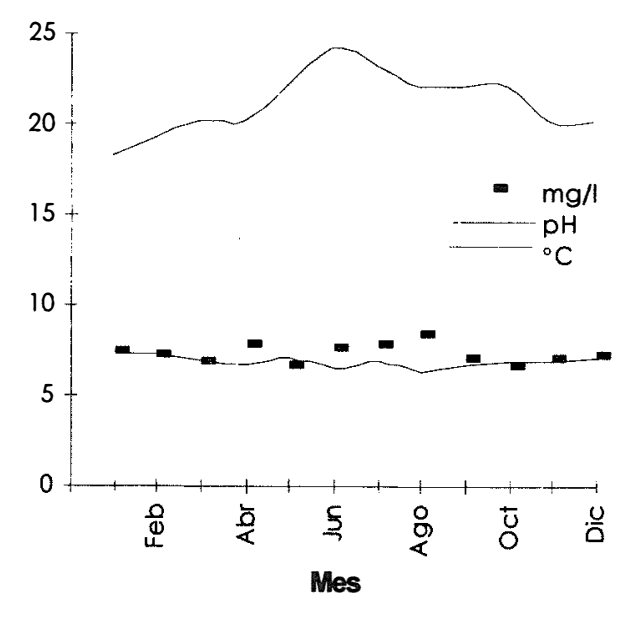

Fig. 1. Parámetros fisico-quimicos del hábitat de Creagrutus brevipinnis, cuenca del rio La Vieja, Alto Cauca, Colombia. 
Alimentación.- C. brevipinnis posee un estómago en forma de saco, redondo; se localiza en la parte anterior de la cavidad celómica en medio de la masa visceral. Se logró determinar que es más largo (promedio $6.56 \mathrm{~mm}$ ) que ancho (promedio $3.79 \mathrm{~mm}$ ); los ciegos pilóricos se hallan en el extremo anterior del estómago, su número varía entre cuatro y seis, siendo cinco el dato mas frecuente. Se determinó que C. brevipinnis mantiene una dieta diversa con predominio de insectos y material vegetal (Cuadro 1). De acuerdo con su aporte a la dieta, los contenidos estomacales se agruparon en 35 categorías troficas ( números 1 al 35) (Cuadro 1) ; los resultados obtenidos según frecuencia de ocurrencia para el total de la población (Cuadro 1) señalan que la oferta alimentaria está dominada por larvas de Diptera (Simuliidae), ninfas de Ephemeroptera y restos de insectos, trozos de frutos de corozo (Arecacea) y semillas de diversas especies. El análisis de las categorías alimentarias a través del método numérico (Cuadro1) indicó la importancia de la selección y captura de organismos de Diptera ( Simuliidae) ( $17.06 \%)$, restos de otros insectos $(7.92 \%)$, Arecaeae $(29.99 \%)$ Bacillariophyceae

\section{CUADRO 1}

Contenido estomacal en Creagrutus brevipinnis

Contenido

Trichoptera

Odonata

Coleoptera

Diptera: Simulidae

Hymenoptera: Formicidae

Ephemeroptera

Lepidoptera

Hemiptera

Isoptera

Orthoptera

Oocitos de Diptera

Restos de otros insectos

Arachnida:Aranea

Nematoda

Annelida

Mollusca:Gastropoda

Frutos de arecaceae

Poaceae

Bacillariophyceae

Semillas

Hojas

Tallos

Fr tos

Flores

Pisces: Siluriformes, Loricariidae

Radios de aletas de pez

Arena

Piedras

Trozo de nylon

Raices de angiospermas

Oocitos de pez

Vidrios

Crus aceae

Mic oforidios

Acarina
Cantidad (\%)

1.17

0.50

0.87

17.06

1.27

2.28

0.20

0.02

0.06

0.06

1.13

7.92

0.10

4.32

0.06

0.08

29.99

0.14

13.23

9.79

1.21

1.70

1.49

0.63

0.02

0.02

0.08

1.94

0.02

1.35

0.10

1.11

0.02

0.02

0.02
Frecuencia (\%) 
(13.23\%) y semillas de diversas especies (9.79\%). No se observaron diferencias cualitativas o cuantitativas en la composición del alimento ingerido entre los períodos secos (eneromarzo, junio-septiembre) y lluvioso ( abril-mayo, septiembre-diciembre). Sin embargo, la actividad alimentaria $(R)$ registró diferencias marcadas; de tal manera los valores más bajos se obtuvieron entre abril-junio y noviembre, mientras las observaciones más altas entre eneromarzo y julio, octubre y diciembre (prueba de $\mathrm{U}$ $(\mathrm{Ts}=3.153 ; \mathrm{P}=0.95$ y un análisis de varianza $\mathrm{P}=$ $19.04 ; \mathrm{P}>0.0001)$. C. brevipinnis no efectúa migraciones de tipo trófico; su actividad se lleva a cabo durante el día (6:00 hr-18:00 hr), localiza la presa visualmente, la captura, se coloca en posición contraria a la corriente de agua e inmediatamente la ingiere. Existe una asociación baja y positiva entre la longitud estándar y el largo del intestino $(r=0.5 ; n=317$, la relación 0.866 , valor mínimo 0.57 , valor máximo 1.01 ). No se halló correlación entre el peso del estómago y la longitud del ejemplar $(r=0.19$, $n=465)$, y con el peso total $(r=0.060 ; n=465)$; lo cual indica que el volumen del alimento ingerido es independiente del tamaño del espécimen. Hay una estrecha relación entre longitud total y peso total $(r=0.748 ; n=465)$ y es significativa $(\mathrm{t}=11.28, \mathrm{P}<0.01, \mathrm{gl} 463)$. El factor de condición (Fig. 2) varió entre un valor mínimo de 0.46 (julio) y un máximo de 1.39 (septiembre), y no se observó asociación con el peso del estómago ni con el índice de voracidad.

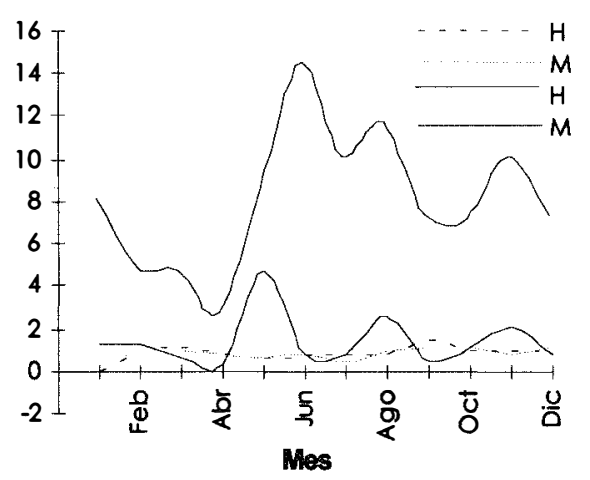

Fig. 2. Índice gonosomático (IGS) y factor de condición (K) para machos (M) y para hembras (H) en Creagrutus brevipinnis, cuenca del río La Vieja, Alto Cauca, Colombia.
Reproducción.- El mayor desarrollo de los ovarios se presentó entre mayo y enero, lo cual abarca tanto el período de lluvias mínimas ("verano") como de lluvias máximas ("invierno"); de febrero a abril decrece el IGS; de manera similar los testículos alcanzaron registros máximos en mayo, agosto y noviembre (Fig. 2 ); además se observaron hembras y machos con gónadas maduras, al igual que larvas y alevines a lo largo del año y en su hábitat característico, lo cual sugiere que $C$. brevipinnis registra un período de desove largo y no efectúa migraciones con fines reproductivos. Durante los meses del estudio no se observó un dominio de uno de los sexos; la diferencia: hembras $203(54.13 \%)$ y machos 172 (45.87\%), no se desvía significativamente de la proporción 1:1 esperada $\left(\mathrm{X}^{2}=2.56\right.$; $\left.\mathrm{P}<0.1 \mathrm{gl}=1\right)$. El pez registra una talla mínima de $3 \mathrm{~cm}$ de longitud estándar en hembras y machos; la talla de maduración para el $50 \%$ de la población fue de 4.0 $\mathrm{cm}$ de longitud estándar (Fig. 3). Durante el transcurso del período del estudio se notó que los machos, a diferencia de las hembras, presentaron espinas en el tercer radio ramificado de la aleta anal, localizadas sobre el borde posterior, a lo largo de la margen media y cuyo número varia entre diez y 13; de igual manera, se observaron espinas entre el segundo y séptimo radio de las aletas ventrales en el borde posterior desde cerca de la base hasta próximo al extremo distal; este carácter se observó en ejemplares sexualmente maduros. Los conteos de ovocitos arrojaron un promedio ponderado de 613 (148-2563) ovocitos por hembra en estado de predesove; no se observó relación entre el número de ovocitos y la longitud estándar, ni con el peso del cuerpo ( $r=0.33$ y 0.54 respectivamente). Las mediciones arrojaron un promedio ponderado para el diámetro del ovocito de $0.6254 \mathrm{~mm}$ (promedio mínimo 0.47 $\mathrm{mm}$; promedio máximo $0.920 \mathrm{~mm}$ ); se encontró una relación inversamente proporcional del diámetro del ovocito con la fecundidad y con la longitud estándar $\quad(r=-0.1638$ y -0.0103 respectivamente). 


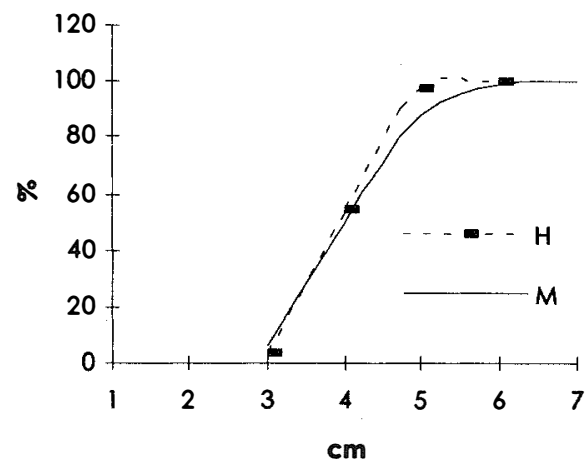

Fig. 3. Porcentajes acumulativos según clases de talla para machos (M) y para hembras $(\mathrm{H})$ en Creagrutus brevipinnis, cuenca del río La Vieja, Alto Cauca, Colombia.
Se determinaron 30 especies de peces que conviven de manera simpátrica con C. brevipinnis (Cuadro 2); se halló que la especie más abundante es Brycon henni (juveniles) (25.83\%); le siguen en orden decreciente de abundancia C. brevipinnis (24.93\%), adultos y juveniles de Chaetostoma fischeri (14.19\%), adultos y juveniles de Sturisoma leightoni (10.40\%), Argopleura magdalenensis (6.50\%) y Bryconamericus caucanus (5.14\%).

\section{DISCUSIÓN}

Wikramanayake (1990) y Wootton (1992) afirmaron que la orientación y forma de la cavidad bucal son de alguna manera atributos

\section{CUADRO 2}

Comunidades de peces en el hábitat de Creagrutus brevipinnis

Especie

Argopleura magdafenensis

Argopleura sp.

Astyanux caucanus

Astyanax microlepis

Branchioica phaneronema

Brycon henni

Bryconamericus caucanus

Cefopsorhamdia boquillae

Bryconamericus sp.

Creagrutus brevipinnis

Chaetostoma fischeri

Characidium caucanus

Hyphessobrycon poecilioides

Lasiancistrus caucanus

Microgenys minutus

Nannorhamdia nemacheir

Poecilia caucana

Rhamdia quelen

Sturisoma leightoni

Priapichthys caliensis

Trichomycterus caliense

Trichomycterus retropinne

Astyanax gisleni

Cariastyanax aurocaudatus

Apteronotus rostratus

Aequidens latifrons

Gephyrocharax caucanus

Roeboides caucae

Oreochromis nilioticus

Homodiaetus sp.

\begin{tabular}{|c|c|}
\hline Cantidad & Frecuenci \\
\hline 115 & 16 \\
\hline 13 & 1 \\
\hline 1 & 1 \\
\hline 20 & 8 \\
\hline 1 & 1 \\
\hline 457 & 15 \\
\hline 91 & 18 \\
\hline 1 & 1 \\
\hline 26 & 5 \\
\hline 441 & 35 \\
\hline 251 & 24 \\
\hline 17 & 8 \\
\hline 6 & 1 \\
\hline 17 & 7 \\
\hline 10 & 5 \\
\hline 6 & 3 \\
\hline 33 & 10 \\
\hline 5 & 3 \\
\hline 184 & 27 \\
\hline 14 & 2 \\
\hline 8 & 4 \\
\hline 2 & 1 \\
\hline 9 & 1 \\
\hline 4 & 1 \\
\hline 1 & 1 \\
\hline 2 & 2 \\
\hline 5 & 1 \\
\hline 27 & 2 \\
\hline 1 & 1 \\
\hline 1 & 1 \\
\hline
\end{tabular}


relacionados con el comportamiento alimentario; $C$. brevipinnis posee una cavidad bucal subterminal o ventralmente orientada, relacionada con su actividad de búsqueda del alimento, la que se efectúa en el sustrato y en la base de la columna de agua, evidente por el contenido estomacal de piedras y fitobentos (Bacillariophyceae). La especie en período de desove ingiere poco alimento, en este lapso se 1ograron observar los únicos tres estómagos vacíos del total examinado; lo cual indica una ingestión previa de alimento con el propósito de obtener reservas energéticas para llevar a cabo su evento reproductivo. La relación longitud estándar-largo del intestino obtenida esta asociada con la eficiencia digestiva y refleja la generalidad de que los peces carnívoros poseen un intestino mas corto que los herbívoros(Kappor et al. 1975; Wikramanayake 1990); además, Bussing (1993) planteó que la relación mencionada en carnívoros es menor de uno, lo cual se cumple con C. brevipinnis, sin embargo, la·morfología de la dentición para carnívoros (dientes alargados y puntiagudos) no coincide con la observada en $C$. brevipinnis. En cuanto a las interacciones tróficas de $C$. brevipinnis se observó que los grupos de más profusión como Brycon henni podrían competir por espacio y por alimento, en esta especie se reportaron datos similares en cuanto a hábitos tróficos (Builes \& Urán 1974); los loricaridos Chaetostoma fischeri y Sturisoma leightoni por su condición de especies alguívoras y betónicas (Power 1984; 1990; López \& RománValencia 1996) se ubicarían en la base de la red trófica del ambiente acuático de $C$. brevipinnis; en estas condiciones es evidente la separación de nichos entre estos taxones, sin embargo, se logró observar fitoplancton en la dieta de $C$. brevipinnis.

Román-Valencia (1995) halló hembras con ovocitos entre enero y febrero; Vazzoler (1996) anoto que el período reproductivo de las especies de peces es diferente, varía entre uno y nueve meses, siendo raras (5-7\%) las que poseen este rango. Harold \& Vari (1994) citaron para C. paralacus la presencia en la aleta anal de espinas entre el primero y el cuarto radio, principalmente en el tercero ramificado, sin embargo, no reportaron este carácter dimorfico sexual en las aletas ventrales. Adebisi (1990) plantea que existe relación amplia entre el diámetro del ovocito y la fecundidad y viceversa, de tal manera, que a mayor diámetro se observa baja fecundidad y viceversa; Heins \& Baker (1987) demostraron que los peces ovíparos producen ovocitos que varían en tamaño, probablemente como una adaptación a una variable ambiental. De modo general Vazzoler (1996) sugirió que la fecundidad varía con la longitud de la hembra, aumenta con el crecimiento y está más relacionada con el tamaño que con la edad del individuo.

\section{AGRADECIMIENTOS}

Este trabajo se llevó a cabo con aportes de la facultad de Investigaciones, Universidad del Quindio (Proyecto 136). Muy agradecido a Germán Arbelaez Serna, Ricardo A. Ferriz, Ana Milena Muñoz, Alejandro Vasquez, Anibal Gonzales y Mario Cardona.

\section{RESUMEN}

Contenido estomacal y aspectos reproductivos fueron estudiados en un pez caracido ( $C$. brevipinnis) de el Alto Cauca (Julio 1995 a diciembre de 1996). La temperatura promedio en la región fue de $21^{\circ} \mathrm{C}$, $\mathrm{pH}$ alrededor de la neutralidad; oxígeno disuelto alto $(8 \mathrm{ppm})$; es una especie no migratoria. El pez es predominantemente insectívora y especializado (Diptera: Simuliidae ; Ephemeroptera), siendo ubicado en el tercer nivel de la red trófica. La actividad alimentaria registró diferencias altamente significativas entre la época lluviosa y la seca de la región $(\mathrm{F}=19.04 ; \mathrm{P}<$ 0.0001 ). El comportamiento alimentario se realizó en el sustrato entre las 6:00 hr y las 18:00 hr. Se observó correlación negativa entre el peso del estómago y el índice de voracidad. La especie en la época de reproducción consumió poco alimento. El desarrollo máximo de las gónadas se registró entre mayo y junio. La fecundidad fue baja ( promedio 613 ovocitos) y el ovocito fue pequeño (promedio 0.6254 $\mathrm{mm})$. La proporción entre sexos fue de $1: 1\left(\mathrm{x}^{2}=2.56 \mathrm{P}<0.1\right.$ $\mathrm{gl}=1$ ) y el tamaño promedio de madurez para la población estudiada fue de $4.0 \mathrm{~cm}$ de longitud estándar. Es simpátrica con 30 especies de peces, siendo La Sabaleta Brycon henni y $C$. brevipinnis las más abundantes 


\section{REFERENCIAS}

Adebisi, A.A. 1990. A mathematical expression for the estimation of relative fish fecundity using oocyte size. Rev. Biol. Trop. 38:323-324.

Anónimo. 1985. Standard methods for examination of water and waste water. Apha, Washington, D.C. 1193 p.

Builes, J. \& A. Uran 1974. Estudio del cíclo sexual de la Sabaleta Brycon henni. Su comportamiento y fecundación artificial. Actual. Biol. 3:2-12.

Bussing, A.W. 1993. Fish communities and environmental characteristics of a tropical rain forest river in Costa $\mathrm{Ri}$ ca. Rev. Biol. Trop. 4: 791-809.

Ferris, A. R. \& W. Salas A. 1996. Dieta de Galaxias maculatus (Jenyns, 1842) (Salmoniformes: Galaxiidae) en un embalse norpatagonico. Boll. Mus. reg. Sci. nat. Torino 14 (1):249-257.

Ghazai, A.M., V. Benech \& D. Paugy. 1991. L' alimentation de Brycinus leuciscus(Teleostei:Characidae) au Mali: sapects qualitifs, quantitatifs at comportamentaux. Ichthyol. Explor. Freshwaters 2: 47-54.

Harold, A.S. , R.P. Vari , A. Machado-Allison \& F. Provenzano. 1994. Creagrutus hysginus (Teleostei:Characiformes) a new species of characid from northeastern Venezuela, Sucre State. Copeia, 1994: 975-979.

Harold, A.S. \& R.P. Vari. 1994. Systematics of the trans Andean species of Creagrutus (Ostariophysi, Characiformes, Characidae). Smithson. Contr. Zool. 551:1-31.

Heins, D.C. \& A. Baker. 1987. Analysis of factors associated with intrasoecific variation in propagule size of a stream dwelling fish p. 223-231 in community and evolutionary ecology of North American stream fishes W.J. Matthewsand, D.C. Heins (Ed.) Univ. of Oklahoma Press Norman, Oklahoma.

Hyslop, E.J. 1980. Stomach contents analysis- a review of methods and their application. J. Fish. 17:411-429.

Kappor, B.G., H. Smith \& L.A. Verighina. 1975. The alimentary canal and digestion in teleost, in F.S. Russell \& M. Younge (Ed.). Advances in Marine Biology 113 : 109-239.
López, J. \& C. Román-Valencia, C. 1996. Sobre la biología del Corroncho Chaetostoma fischeri (Pisces: Loricariidae) en el río La Vieja, Alto Cauca, Colombia. Boletín Ecotrópica 30: 37-57.

Movillo, J. \& N. Bahamonde. 1971. Contenido gastrico y relaciones troficas de Thrsites atun (Euphrasen) en San Antonio, Chile. Bol. Mus. Nat. Hist. XXIX :289-338.

Ortaz, M. 1992. Hábitos alimenticios de los peces de un Río de Montaña Neotropical. Biotropica 24:550-559.

Power, M. 1984. Habitat quality and the distribution of algae grazing catfish in a Panamian stream. Journal of Animal Ecology 53:357-374.

Power, M. 1990. Benthic turfs vs floating mate of algae in river food webs. Oikos 58:67-79.

Román-Valencia, C. 1995. Lista anotada de los peces de la cuenca del río La Vieja, Alto Cauca, Colombia. Boletín Ecotrópica 29: 1-19.

Roman-Valencia, C. \& Cala, P. 1997. Las especies Colombianas del genero Creagrutus (Pisces,Characidae). Rev. Acad. Colomb. Cienc. XXI: 141-153.

Saul, G.W. 1967. An Saul ecological study of fishes at site in upper Amazonian Ecuador. Thesis M.Sc. The University of Kansas, Lawrence, Kansas, USA 113 p.

Vari, R.P. ,A.S. Harold, C.A. Lasso \& A. Machado-Allison. 1993. Creagrutus lepidus, a new species from the Rio Aroa Systems, Yaracuy State, Venezuela (Teleostei: Characiformes, Characidae). Ichthyol. Explor. Freshwaters 4: 351-355.

Vazzoler, A.E.A. de M. 1996. Biologia da reproducao de peixes teleosteos: teoria e pratica. EDUEM, Maringa, Sao Pablo 169 p.

Wikramanayake, D.E. 1990. Ecomorphology and biogeography of a tropical stream fish assemblage: evolution of assemblage structure. Ecology 7 :1756-1764.

Wootton,, R. J. 1992. Fish Ecology. Chapman \& Hall, Nueva York, $221 \mathrm{p}$. 